and established a clinical network. This provides a strong basis on which the commissioning team can take forward an option appraisal process.

\section{THE TRIGGER PROJECT: INTRODUCING ELECTRONIC PATIENT REPORTED OUTCOME MEASURES INTO RADIOTHERAPY SERVICES}

${ }^{1} \mathrm{~A}$ Macnair* ${ }^{1} \mathrm{~A}$ Sharkey, ${ }^{2,3} \mathrm{~K}$ Le Calvez, ${ }^{4} \mathrm{R}$ Walters, ${ }^{5} \mathrm{~L}$ Smith, ${ }^{6} \mathrm{~A}$ Nelson, ${ }^{7,8} \mathrm{~J}$ Staffurth, ${ }^{3,9} \mathrm{M}$ Williams, ${ }^{10} \mathrm{D}$ Bloomfield, ${ }^{11} \mathrm{~J}$ Maher, Trigger Project Collaborative group. ${ }^{1}$ Macmillan Cancer Support and Royal College of Radiologists, Royal college of Radiologists, London; ${ }^{2}$ Charing Cross Hospital, Radiotherapy Department, London; ${ }^{3}$ Computational Oncology Laboratory, Institute of Global Health Innovation, Faculty Building, South Kensington Campus, Imperial College, London; ${ }^{4}$ Pelvic Radiation Disease Association, Epsom; ${ }^{5} \mathrm{NHS}$ England National Cancer Programme, London; ${ }^{6}$ Marie Curie Research Centre, Division of Population Medicine, School of Medicine, Cardiff University, Cardiff; ' Velindre University NHS Trust, Cardiff; ${ }^{8}$ School of Medicine, Cardiff University, Cardiff; ${ }^{9}$ Charing Cross Hospital, Radiotherapy Department, London; ${ }^{10}$ Brighton and Sussex University Hospitals NHS Trust, Brighton: ${ }^{11}$ Macmillan Cancer Support, London

10.1136/leader-2019-FMLM.110

Patients receiving pelvic radiotherapy can experience long term GI side effects post-radiotherapy. The Trigger project identifies patients experiencing symptoms of radiation-related bowel toxicity using the ALERT-B questionnaire, and directs them to the appropriate clinician. Trigger is a service evaluation project, aiming to prove the utility of electronic PROMs, and to demonstrate the feasibility of a low-resource project as a model for collecting PROMs. It is a collaboration between Macmillan Cancer Support, the Royal College of Radiologists, and three NHS Trusts: Velindre, Imperial College Healthcare and Brighton and Sussex University Hospitals.

Patients register on the Trigger website, hosted by My Clinical Outcomes, and receive periodic emails to complete the short ALERT-B questionnaire electronically, to screen for longterm bowel symptoms which could have been caused by pelvic radiotherapy. If answering 'yes' to any of the questions, patients are directed to appropriate services. 6 months following the completion of their radiotherapy, patients are sent a separate questionnaire to evaluate the utility of the project.

336 patients registered in first the 9 months across the 3 sites. Patients with a range of different cancers signed up: anal (2\%), bladder (1\%), prostate $(87 \%)$, rectal $(4 \%)$ and gynaecological (6\%). 43 patients (/65 (uptake 66\%)) have answered their 6-month post treatment questionnaire, and $72 \%$ answered 'yes' to at least one of the ALERT-B questions. $85 \%$ of responding patients reported they found the Trigger project helpful.

These promising results show that electronic PROMS can be introduced in radiotherapy departments using a low resource model. The Trigger project works as a feasibility model, showing patients engage with electronic PROMs projects, and find them useful. PROMs for other tumour types could be collected in a similar manner, based on the lowresource model used here, using site-specific PROMs based on the ALERT-B tool.

\section{MEDISENSE TECHNOLOGY FOR BREAST CANCER}

Aswini Misro, Vikash Sharma. Kings College NHS London, UK and University of Hull, UK

10.1136/leader-2019-FMLM.111
Introduction It is not uncommon for a patient coming to the NHS to present with investigations and treatments done in EU countries and outside the EU for various medical and surgical conditions. Many times, reports are written in languages other than English and therefore does not to contribute to medical consultations.

Another issue with the patients is that they feel helpless and frustrated when they can't understand what the reports mean for them. To resolve these problems, we developed a technology called Medisense is a medical report image capture, data extraction, validation and interpretation technology. By using this technology, doctors and patients can take a snap of the picture of any report and get an instant translation, interpretation and review of the report.

Study design In the current study, we carried out 97 cases of breast cancer histopathology reports of various patients. The test was carried out using a specified protocol in a test environment.

Results The study results show that in all the 97 cases, the image capture was successful. However, the data extraction was successful only in 93 cases. In 90 cases, the data interpretation was correct. In the 4 instances where image capture was not successful, the poor printing quality of the report $(n=1)$, folds and creases in the report $(n=1)$, and technical error $(\mathrm{n}=2)$ was responsible.

Conclusions Medisense will be a helpful tool in medical consultations when a patient arrives with a report in a nonnative language. This would also help patients interview and interpretation of their medical reports. Further studies and improvements are required to optimise this technology further.

\section{NEW RAPID ACCESS UROLOGY CLINIC}

${ }^{1}$ Emily Simon Thomas, ${ }^{2}$ Rachel Hawthorne, ${ }^{3}$ Ayah Babiker, ${ }^{2}$ Kai Chi Chan. ${ }^{1}$ Oxford Health NHS Foundation Trust; ${ }^{2}$ Oxford University Hospitals NHS Foundation Trust; ${ }^{3}$ Buckinghamshire Health NHS Foundation Trust

\subsection{6/leader-2019-FMLM.112}

Rapid access urology clinics had been trialled elsewhere in the country with variable success. ED, urology consultants and registrars, and senior nurses (i.e. the stakeholders who would be directly impacted by this intervention) were involved in the decision to convert two side rooms to such a clinic in our DGH. The aim was to see non-elective urology patients efficiently to ensure prompt management and avoid admission where possible.

The proposal and initial data were discussed in the monthly urology governance meetings. The pilot started in August 2018. Patients could be referred by ED, GP, other hospital departments via the on call urology registrar or selfrefer. Patients discharged from the urology ward were informed about this, as were ED staff, GPs, and other departments such as radiology and oncology. Feedback from patients and ED staff were welcomed and outcomes measured.

Data was collected prospectively during an initial 47-day pilot period and the subsequent three months. Each attendance was reviewed individually. The hot clinic saw 107 patients in the pilot period and 217 in the subsequent three months. ED avoidance was estimated at $61 \%$ in the trial 